\title{
Magnetoelastic Nature of Ferromagnetic Shape Memory Effect
}

\author{
V. A. Chernenko $0^{1,2, a}$ and V. A. L'vov' \\ ${ }^{1}$ Institute of Magnetism, Vernadsky str. 36-b, Kyiv 03142, Ukraine \\ ${ }^{2}$ CNR-IENI, C.Promessi Sposi, 29, Lecco 23900, Italy \\ ${ }^{3}$ Dept. Radiophysics, Taras Shevchenko University, Glushkov Str. 2, bld.5, Kyiv 03022, Ukraine \\ avvladimir.chernenko@gmail.com, bvictorlvov@univ.kiev.ua
}

\begin{abstract}
Keywords: Ferromagnetic shape memory effect, Ni-Mn-Ga martensite, magnetostrictive mechanism, lattice instability
\end{abstract}

\begin{abstract}
The giant magnetically-induced deformation of ferromagnetic shape memory alloys results from the magnetic field-induced rearrangement of twinned martensite under the magnetic field. This deformation is conventionally referred to as the magnetic-field-induced-strain (MFIS). The MFIS is comparable in value with the spontaneous deformation of crystal lattice during the martensitic transformation of an alloy. Although the first observations of MFIS were reported more than 30 years ago, it has got a world-wide interest 20 years later after the creation of the $\mathrm{Ni}-\mathrm{Mn}-\mathrm{Ga}$ alloy system with its practically important room-temperature martensitic structure and experimental evidence of the large magnetostriction. The underlying physics as well as necessary and sufficient conditions for the observation of MFIS are the main focus of this chapter. A magnetostrictive mechanism of the unusual magnetic and magnetomechanical effects observed in Ni-Mn-Ga alloys is substantiated and a framework of consistent theory of these effects is outlined starting from the fundamental conception of magnetoelasticity and the commonly known principles of ferromagnetism and linear elasticity theories. A reasonable agreement between the theoretical deductions and available experimental data is demonstrated and, in this way, a key role of magnetoelastic coupling in the magnetomechanical behavior of $\mathrm{Ni}-\mathrm{Mn}-\mathrm{Ga}$ alloys is proved. A correspondence of magnetostrictive mechanism to the crystallographic features of MFIS and the basic relationships of the thermodynamics of solids are discussed.
\end{abstract}

\section{Introduction}

Both the spontaneous ferromagnetic ordering and/or externally stimulated magnetization process in solids are always accompanied by volume and shape changes, which are termed as a magnetostriction [1]. Magnetostriction manifests itself as the magnetically-induced elastic straining of the experimental specimen. The magnetically ordered solids show typical values of the magnetoelastic strain $\varepsilon^{(m e)}$ of about $10^{-6}-10^{-5}$ while the strain values inherent to the practically used magnetostrictive materials belong to the interval $10^{-4}-10^{-3}$. Magnetostriction arises because the intensity of spin exchange between electrons and the force of spin-orbit interaction depend on the spatial positions of atoms in the crystal lattice. Thus, these dependencies can be considered at the origins of isotropic and anisotropic spin-lattice interactions.

The magnetically induced volume/shape changes have a great impact on both magnetic and elastic properties of a large number of solids. Well-known examples are Invar alloys with a large spontaneous volume magnetostriction or MnAs-type compounds exhibiting lattice distortion and large volume effect at the Curie temperature. Due to the localized nature of $f$-electrons in atoms, the spin-lattice interactions in the rare-earth elements and compounds are especially strong giving rise to the famous phenomenon of giant magnetostriction (TERFENOL-D).

Curiously, the large magnetostriction of single crystalline $\mathrm{Dy}$ and $\mathrm{Tb}$, which was found in 1970 s, proved to be accompanied by a giant magnetoplastic deformation $\left(\varepsilon \approx 4 \times 10^{-2}\right.$ in the field around $10 \mathrm{~T})$. This phenomenon was explained by a magnetic field-induced mechanical twinning 
[2]. Scientifically sound results, which were obtained for $\mathrm{Dy}$ and $\mathrm{Tb}$, did not receive much attention due to the obvious lack of practical importance: the magnetoplastic deformation was observed at 4.2 $\mathrm{K}$ and under very high magnetic fields.

On the other hand, the ferromagnetic shape memory alloys, such as $\mathrm{Fe}-\mathrm{Pd}$ (30 at.\%), $\mathrm{Fe}_{3} \mathrm{Pt}$ and $\mathrm{Fe}-\mathrm{Ni}-\mathrm{Co}-\mathrm{Ti}$, which are Invars exhibiting thermoelastic martensitic transformations (MTs), have been known for over 30 years (see, e.g., [3-5]). Particularly, a magnetic field influence on the martensitic transformation temperatures has been studied in these alloys (see e.g., [6-9]). The obtained results pointed to the possibility of a magnetic-field-induced MT with a certain hysteresis and characteristic strain (either volumetric or deviatoric) at a constant temperature above the MT temperature. After obtaining experimental confirmation, this MT was tentatively termed as the magnetoelastic effect [9]. Such an idea and correspondent research line have led, later on, to the development and studies of the magnetostrained materials where the MT temperature is artificially merged with the Curie temperature [10-13] and/or natural materials such as, e.g., MnAs [14], FeRh [15] or $\mathrm{TbMn}_{2}$ [16] exhibiting spontaneous magnetostructural transformation on cooling. In all these materials, the strain usually recovers after the removal of the magnetic field.

Furthermore, it is interesting to note that the martensitic Invars showing a strong negative slope of MT temperature as a function of hydrostatic pressure appeared to be a model object to demonstrate the baroelastic effect: the sample, which was preliminary deformed in the martensitic state, recovers its shape through the pressure-induced reverse MT transformation [17]. The magnetic analog of this effect was recently realized by using Mn-rich Heusler compounds, which show the magnetic field-induced shape recovery in the course of reverse MT accompanied by the metamagnetic phase transition [18].

Before the end of $1980 \mathrm{~s}$, some of the stoichiometric ferromagnetic $\mathrm{X}_{2} \mathrm{YZ}$ Heusler compounds, where $\mathrm{X}$ is $3 \mathrm{~d}$-metal, $\mathrm{Y}$ is usually Mn but could be other $3 \mathrm{~d}$-metal or rare-earth elements, $\mathrm{Z}$ is one of the elements from groups III or IV of the periodic table, have been already known to exhibit a lattice instability towards a martensitic transformation (see, e.g., $[19,20]$ and references therein). Upon an analogy with Cu-base shape memory alloys (SMAs), such martensitic Heusler alloys were later considered as Hume-Rothery alloys exhibiting a Zener-type lattice instability [10]. Particularly, the low-temperature MT and the formation of martensitic plate-like morphology in $\mathrm{Ni}_{2} \mathrm{MnGa}$ compound (both features are the prerequisites of the thermoelastic MT) were described in 1984 [20]. This information, together with the long-term experience with thermoelastic martensitic transformations in the Fe-base Invar alloys, prompted us to verify experimentally the thermoelastic nature and related shape memory and superelastic properties of this compound in 1989 [21].

Furthermore, the enormous shift of MT temperature towards the high temperatures was found by variations of alloy composition beyond stoichiometry, which has led to the creation of the family of non-stoichiometric Ni-Mn-Ga ferromagnetic shape memory alloys (FSMAs) with practically attractive MT temperatures, which are close to the room temperature $[22,23]$.

The magnetostriction measurements of one of the $\mathrm{Ni}-\mathrm{Mn}-\mathrm{Ga}$ single crystals listed in Ref.[23] were carried out in 1996 by two research groups using pulse [24] and quasi-static [25] magnetic fields. An abnormally large magnetostriction of about $0.2 \%$ was measured in the latter case. It was explained by the magnetic field-induced twin boundary motion [25]. This mechanism of magnetic field-induced strain (MFIS) differs from all the aforementioned mechanisms because it proceeds in the martensitic phase and comprises a field-induced conversion of one twin component into another, the process being inherently dependent on the mutual orientation of the magnetic field and hard magnetic directions of twin components.

As follows from the diagram in Fig.1, the world-wide research/publication boom on MFIS in $\mathrm{Ni}-\mathrm{Mn}-\mathrm{Ga}$ materials started three years later, after 1996. As a result, the record-breaking reversible magnetostrain values of $6 \%$ and $10 \%$ were achieved experimentally as a consequence of the large magnitudes of the tetragonal or orthorhombic distortions of crystal lattice in the martensitic phase, respectively [26-29]. Fig. 1 and the available review articles [13,30-37] demonstrate that Ni-Mn$\mathrm{Ga}$ alloys are the most intensively studied FSMA systems so far. 


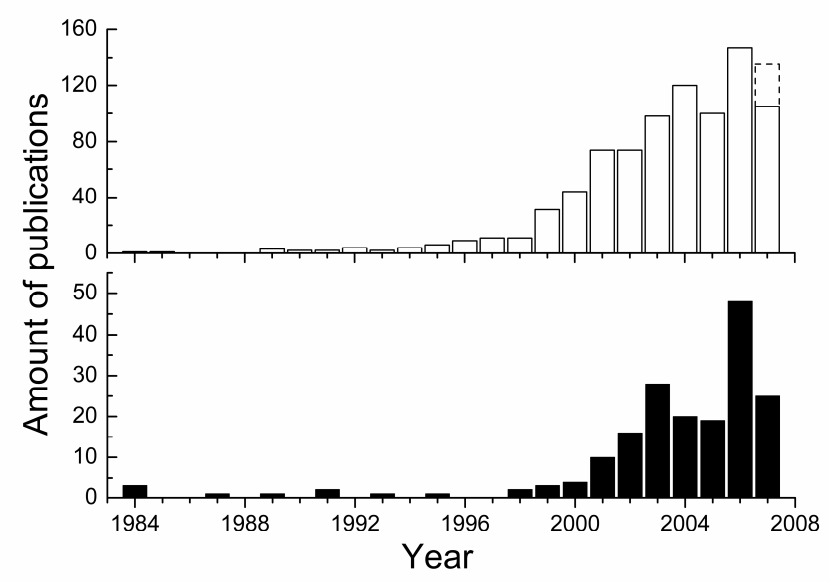

Fig.1. Dynamics of the annual amount of publications devoted to Ni-Mn-Ga FSMAs (white columns) and alternative FSMA systems (black columns). The expected amount of delayed publications is marked by dash.

The amount of publications displayed in Fig.1 as the black columns are related to the other FSMA systems such as Fe-Pd [31,38,39], Fe 3 Pt [31,39], Ni-Mn-(Al, Sn, Sb, In) [18,40-43], Co-Ni(Al, Ga) [44-48] and Ni-Fe-(Al,Ga) [49-53]. The black part of the diagram in Fig.1 and overview articles $[54,55]$ prove an increasing interest towards the alternative systems as well.

The best-working room-temperature easy-axis Ni-Mn-Ga FSMAs and the phenomena which they exhibit continue to be the scope of intense fundamental and applied research nowadays. The aforementioned record-breaking values of magnetostrain, the high values of estimated magnetocaloric effect [56-58], and the effect of twinning-strain-induced rotation of the magnetization vector [59-61] are the phenomena in the focus of current research and development.

A highlight of the necessary and sufficient conditions of MFIS as well as the analysis of the underlying physics are the main subject matters of this chapter. The lattice instability in $\mathrm{Ni}-\mathrm{Mn}-\mathrm{Ga}$ FSMAs is described as the main prerequisite of MFIS. Thermodynamic arguments are used to remove an apparent gap between the physics of ordinary magnetostriction and large MFIS. A magnetostrictive mechanism of the unusual magnetic and magnetomechanical properties of $\mathrm{Ni}-$ $\mathrm{Mn}-\mathrm{Ga}$ alloys is substantiated and a consistent theory of these effects is developed starting from the fundamental conception of magnetoelasticity and the commonly known principles of the theory of ferromagnetism. This approach to comprehension of MFIS was already formulated by the authors and their co-workers in the past as a magnetoelastic model. The microscopic features of ferromagnetic martensite are demonstrated to be in line with a magnetoelastic model.

\section{Structural instability and magnetostriction}

One might say that an inherent lattice instability towards thermoelastic MT $[10,20]$ is a primary reason explaining the ability of the ferromagnetic $\mathrm{Ni}-\mathrm{Mn}-\mathrm{Ga}$ alloys to exhibit the giant MFIS. This instability is mainly related to the uniform (110)[1 $\overline{1} 0]$ shear often accompanied by shuffling in the same system. It belongs to a Zener-type instability typical of $\beta$-alloys (see $[10,62]$ and references therein) when a parent phase with bcc open lattice transforms during cooling into a lower-symmetry close-packed martensitic phase characterized by the invariant-plane Bain deformation. A Zenertype instability implies a low-restoring force towards aforementioned shear and high entropy of cubic phase due the low-lying phonon modes. In order to accommodate large Bain strains exhibited by the differently oriented martensitic variants, secondary invariant-lattice strains occur involving the same shear system so that a twinned microstructure is formed. 
It is of a great significance that the aforementioned shear in Ni-Mn-Ga intermetallics, as well as in other $\beta$-alloys [62] and In-Tl [63] or Fe-Pd alloys [64], is characterized by a low restoring force, i.e. their shear elastic modulus $C^{\prime}=\left(C_{11}-C_{12}\right) / 2$ is very small. Moreover, $C^{\prime}$ demonstrates a wellpronounced softening of these alloys on cooling in the cubic phase and reaches a minimum of about a few GPa near MT [65,66]. Fig. 2(a) illustrates a typical temperature evolution of the lowfrequency elastic modulus for the single crystalline $\mathrm{Ni}_{2} \mathrm{MnGa}$ sample on cooling [65]. The resonance ultrasonic measurements of the polycrystalline samples show similar temperature

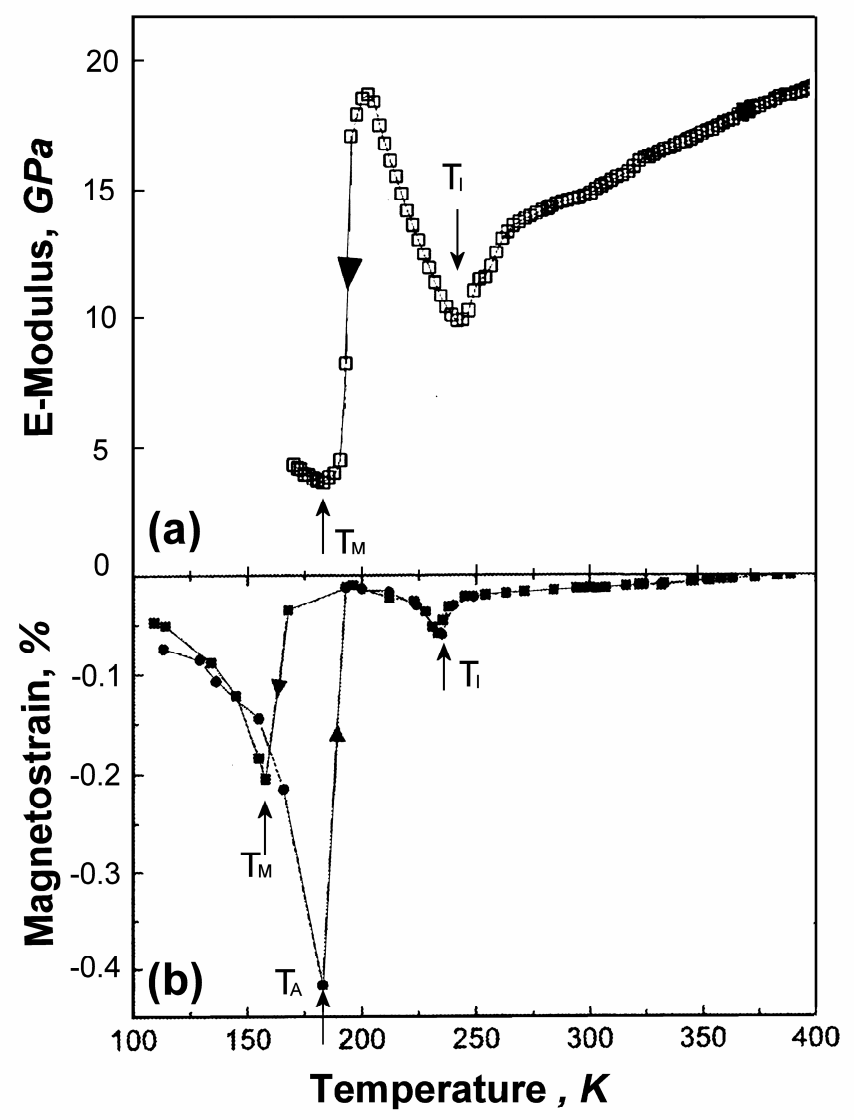

Fig.2. Temperature dependence of the elastic modulus on cooling (a) and coolingheating curves of longitudinal magnetostriction in the field of $5 \mathrm{kOe}(\mathrm{b})$ for two $\mathrm{Ni}_{2} \mathrm{MnGa}$ single crystals of close compositions [65,70,71]. $T_{M}$ and $T_{A}$ are characteristic temperatures of the forward and reverse martensitic transformations, respectively; $T_{I}$ is the temperature of premartensitic transformation into the intermediate phase.

dependence in shear modulus as in Fig. 2(a) [67]. The two minimums of modulus and magnetostriction in Fig. 2 are associated with the premartensitic, forward and reverse martensitic transformations at $T_{I}, T_{M}$ and $T_{A}$ temperatures, respectively. The lattice instability of the parent phase arises when approaching the premartensitic transformation temperature $T_{I}$. The premartensitic transition is preceded by an almost linear decrease of the elastic modulus reflecting the precursor events. According to Fig. 2(a), the total decrease of this modulus in the cubic phase is about 30-40\%. The premartensitic transition is associated with the soft 1/3[110]TA $\mathrm{T}_{2}$-phonon condensation (energy of the whole $\mathrm{TA}_{2}$ branch lowers during cooling as well) [65-69]. The modulus stiffening in the premartensitic phase (Fig.2a) slows down but does not prevent the crystal lattice to be globally unstable towards the mechanism of its uniform distortion during MT. This fact seems to indicate that the martensitic transformation is not directly controlled by a phonon mechanism of instability. The MT may be rather a consequence of the instability of electronic subsystem and the lattice anharmonicity (see, e.g., [13,20,65]). It is well known that in view of the low values of second-order shear modulus (usually measured under the assumption that Hook's law is valid, which is not strictly true), the higher order elastic modules play an important role in the occurrence of MT. 
It is clearly seen in Fig.2 that one of the consequences of a reduced modulus, especially in the temperature range of structural transformations, is a considerably enhanced ordinary magnetostriction characterized by the magnetoelastic strain $\varepsilon^{(m e)}$. For instance, at the parent-tointermediate phase transition in Fig. 2(b) it is about $0.06 \%$ (the measurements were carried out in a cyclic magnetic field [70,71]). According to the expression $\varepsilon^{(m e)} \approx 2 \delta_{1} / C^{\prime}$ [70], where $\delta_{1}$ is the magnetoelastic parameter, the occurrence of the right-hand-side minimum in the curve of Fig.2(b) is described by the concurrent minimum of elastic modulus in Fig.2(a).

In the temperature range of MT, the cyclic magnetostrain is much larger, up to $0.4 \%$ $\{$ Fig.2(b)\}. This value implies a predominant contribution of the reversible magnetic-field-induced motion of twin boundaries operated by the twinning dislocations, as it cannot be explained simply by an ordinary magnetostriction. A reverse motion of some of the twinning dislocations is possible under the action of local internal stresses developed during their forward pass under magnetic field or external stress $[36,37,72,73]$.

The fascinating feature of $\mathrm{Ni}-\mathrm{Mn}-\mathrm{Ga}$ alloys in single-crystalline form is a combination of abnormally low values of elastic modulus and extremely high mobility of twin boundaries. In our opinion this combination is the main factor, which must be taken into account to explain the very low values of twinning stress (about few MPa) estimated from the experimental stress-strain dependencies in the martensitic state $[28,59,73,74]$. This twinning stress could be smaller than the equivalent mechanical stress (magnetostress) produced by a magnetic field. Whenever this condition holds, it is sufficient for the occurrence of the giant MFIS. The magnetoelastic mechanism of MFIS and the role of magnetostriction in the magnetic properties of ferromagnetic shapememory alloys are explained in the next sections.

\section{Magnetoelastic model of magnetostrain effect}

This section is devoted to an application of the consistent phenomenological theory of magnetoelasticity (see, e.g., [75,76]) to the ferromagnetic Ni-Mn-Ga alloys. Evidences are given below that the magnetic and magnetomechanical properties of these magnetostrictive alloys are governed by the magnetoelastic interactions inherent to all magnetic solids. In particular, a magnetoelastic origin of MFIS is substantiated. It is demonstrated that the macroscopic models of MFIS, which disregard the theory of magnetoelastic interactions (explicitly set forth in Refs.[75,76]), are self-contradictory and incompatible with the fundamental principles of linear elasticity of solids.

Preliminary considerations. The MFIS is related to a transformation of the specimen microstructure in an increasing magnetic field. In accordance with existing experimental data, the microstructure of single crystalline specimens exhibiting MFIS can be modeled by alternating domains (variants) of tetragonal crystal lattice, which form a sequence of crystallographic twins (Fig. 3).

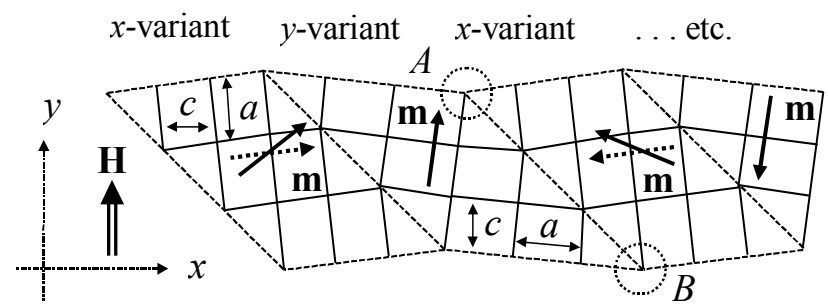

Fig. 3. Schematic representation of twotwin-variants configuration in a tetragonal ferromagnetic martensite.

Let the coordinate axes be aligned with $\langle 100\rangle$ crystallographic directions and the magnetic field be applied in $y$ direction. Field application breaks the equivalence of twin components, and hence, initiates the growth of the volume fraction of $y$-variant to the expenses of the $x$-variant. In this way it induces the deformation of the specimen. An absolute value of the appropriate strain tensor 
component may be estimated as $\varepsilon \sim(1-c / a) / 2$, where $c$ and $a$ are short and long lattice parameters of the tetragonal unit cell shown in Fig.3. Moreover, if the magnetic field is applied along the hard direction ( $a$-axis) of the one-variant specimen, it will cause its twinning and subsequent detwinning with a final formation of a one-variant specimen, but with easy direction $(c$ axis) aligned with the magnetic field. In this case, the observed deformation is close to the theoretical limit $(1-c / a)$.

The large value of magnetostriction constant $\lambda \approx 1.3 \cdot 10^{-4}$ was reported and the reversible magnetostrictive deformation $\varepsilon^{(m e)} \approx-0.01 \%$ was observed for the cubic (austenitic) phase of Ni$\mathrm{Mn}-\mathrm{Ga}$ alloy in a saturating magnetic field [25], see also Fig.2. On the other hand, the compressive stress $\sigma \approx 1.75 \mathrm{MPa}$ applied along one of the $\langle 100\rangle$ crystallographic directions of the single-variant martensite is sufficient for the accomplishment of the twinning-detwinning cycle [73]. The abnormally low value of the shear elastic modulus $C^{\prime} \sim 5 \mathrm{GPa}<<\mathrm{C}_{11}$ reported for the cubic phase of a number of $\mathrm{Ni}-\mathrm{Mn}-\mathrm{Ga}$ alloys $[65,77]$ results in the following estimations for the Young modulus $E$ and the elastic strain $\varepsilon^{(f)}$, needed for the accomplishment of the twinning-detwinning process:

$$
\begin{aligned}
& E \equiv \frac{2 C^{\prime}\left(C_{11}+2 C_{12}\right)}{C_{11}+C_{12}} \approx 3 C^{\prime}, \\
& \varepsilon^{(f)} \sim \sigma / E \sim-10^{-4}=-0.01 \% .
\end{aligned}
$$

Thus, the elastic strain, which is induced by the detwinning stress, is numerically close to the magnetostrictive deformation of the cubic phase.

It may be assumed that the magnetostriction of the tetragonal phase is close in value to the magnetostriction of the cubic phase. In this case, the magnetostrictive deformation of twinned crystal lattice would exceed the value needed for the start of twining-detwinning process when the increasing magnetic field exceeds some threshold value. In other words, the magnetostrictive deformation triggers the twinning-detwinning process, and so, a magnetoelastic interaction is the physical origin of the giant MFIS observed in Ni-Mn-Ga alloys. This idea was first formulated in Refs. [78-81] and verified by the qualitative and quantitative comparison of theoretical results with experimental data [73, 82-84].

Arguments will be given that the magnetoelastic model of MFIS is compatible with the fundamental crystallographic concepts of martensitic transformation, such as a Wechsler -Liberman - Read theory [85]. According to these concepts, the spatial orientation of twin boundaries can be definitely calculated from the values of lattice parameters. Small changes in these parameters result in an appreciable reorientation of the boundaries.

It will be clarified below that the magnetoelastic model is compatible with the microscopic mechanism of MFIS studied in $[36,37,86]$.

Model formulation. The cubic-tetragonal martensitic transformation is characterized by the diagonal components of strain tensor $\varepsilon_{i i}$ and so, the non-diagonal components may be disregarded. According to the commonly recognized theory of magnetoelasticity [75,76], the free energy $F$ is the sum of elastic, magnetic and magnetoelastic energies denoted as $F_{e}, F_{m}$ and $F_{m e}$, respectively. Thus,

$$
F\left(\varepsilon_{i i}, \mathbf{M}, \mathbf{H}\right)=F_{e}\left(\varepsilon_{i i}\right)+F_{m}(\mathbf{M}, \mathbf{H})+F_{m e}\left(\varepsilon_{i i}, \mathbf{M}\right),
$$

where $\mathbf{M}$ is the magnetization vector and $\mathbf{H}$ is the magnetic field applied to the specimen.

In the harmonic approach, the expression for the elastic energy of the cubic phase is:

$$
F_{e}=3\left(C_{11}+2 C_{12}\right) u_{1}^{2} / 2+C^{\prime}\left(u_{2}^{2}+u_{3}^{2}\right) / 6
$$


where $C_{11}, C_{12}$ and $C^{\prime}=\left(C_{11}-C_{12}\right) / 2$ are elastic modules,

$$
u_{1}=\left(\varepsilon_{x x}+\varepsilon_{y y}+\varepsilon_{z z}\right) / 3, \quad u_{2}=\sqrt{3}\left(\varepsilon_{x x}-\varepsilon_{y y}\right), \quad u_{3}=2 \varepsilon_{z z}-\varepsilon_{y y}-\varepsilon_{x x} .
$$

The magnetic energy of the cubic phase is expressed as:

$$
F_{m}=J y^{2} / 2+M^{2}(\mathbf{m} \cdot \mathbf{D} \cdot \mathbf{m}) / 2-\mathbf{m H} M
$$

where the first, second and third terms are the exchange, magnetostatic and Zeeman energies respectively, $J$ is the spin exchange parameter, $\mathbf{D}$ is demagnetization matrix, the dimensionless variables $y=M(T) / M(0)$ and $\mathbf{m}=\mathbf{M}(T) / M(T)$ characterize the absolute value and direction of the magnetization vector, respectively [82].

The expression for the magnetoelastic energy of the cubic phase has the form:

$$
F_{m e}=-\delta_{0} y^{2} u_{1}-\delta_{1}\left[\sqrt{3}\left(m_{x}^{2}-m_{y}^{2}\right) u_{2}+\left(2 m_{z}^{2}-m_{y}^{2}-m_{x}^{2}\right) u_{3}\right] \text {, }
$$

where $\delta_{0}$ and $\delta_{1}$ are the magnetoelastic energy parameters characterizing the isotropic and anisotropic parts of the spin-lattice interaction in the cubic phase, respectively [78,87]. Strictly speaking, the anisotropic spin-lattice interaction in the tetragonal phase is characterized by two magnetoelastic parameters, but the difference in these parameters is of the order of $\delta_{1}(1-c / a)<<\delta_{1}$. The energy terms of the fourth order in the magnetic vector and of the second order in the strain tensor components are comparatively small, and therefore, are omitted in Eqs.5 and 6.

The cubic-tetragonal MT transformation in $\mathrm{Ni}_{2} \mathrm{MnGa}$ single crystals results in the spontaneous deformation of the cubic lattice, which is characterized by the strains $u_{2,3}^{M} \sim c / a-1 \approx-0.05$. Let the deformation of the stressed tetragonal lattice be counted off the spontaneous value, i.e. $u_{\alpha} \rightarrow u_{\alpha}^{M}+u_{\alpha}, \alpha=1,2,3$. By virtue of the fairly small values of spontaneous strains, Eq. 2 for the elastic energy is approximately valid for the tetragonal lattice, i.e. $F_{e}^{(t)} \approx c o n s t+F_{e}$ as long as $u_{\alpha}^{M}$ values are constant. The magnetic energy Eq. 5 does not depend on strains, and therefore, $F_{m}^{(t)}=F_{m}$. Finally, using the condition $\mathbf{m}^{2}=1$, the magnetoelastic energy of the $j$-variant of the tetragonal phase can be expressed as:

$$
F_{m e}^{(t)}=-\delta_{0}(\Delta V / V) y^{2} / 3-K_{u} m_{j}^{2}+F_{m e},
$$

where $\Delta V / V$ is the fractional change in specific volume of the specimen; magnetic anisotropy constant:

$$
K_{u}=6 \delta_{1}(c-a) / a
$$

is positive for the $\mathrm{Ni}-\mathrm{Mn}-\mathrm{Ga}$ martensites with $c<a$, because the fourfold symmetry axis is parallel to the direction of easy magnetization. Hence, magnetoelastic parameter $\delta_{1}$ is negative. For more details concerning Eq. 7 and its consequences see Refs. [78,82,88].

According to the fundamental principle of the Thermodynamics of solids, the equilibrium values of elastic strains correspond to the minimum of Gibbs potential:

$$
G=F-\left(\sigma_{1} u_{1}+\sigma_{2} u_{2}+\sigma_{3} u_{3}\right) / 6,
$$

where 


$$
\begin{aligned}
& \sigma_{1}=\left(\sigma_{x x}+\sigma_{y y}+\sigma_{z z}\right) / 3, \quad \sigma_{2}=\sqrt{3}\left(\sigma_{x x}-\sigma_{y y}\right), \\
& \sigma_{3}=2 \sigma_{z z}-\sigma_{y y}-\sigma_{x x} .
\end{aligned}
$$

Hence, the equilibrium values of strain tensor components satisfy the conditions:

$$
\partial G / \partial u_{\alpha}=0 \text {. }
$$

Conditions expressed by Eq.11 result in the generalized Hooke's law establishing interrelation between strains and stresses. It says that every stressed state of a solid is elastically deformed and every elastically deformed state is stressed. In particular, for $\alpha=2$, Eq.11 results in the relationship:

$$
u_{2}=\left(\sigma_{2}^{(m e)}+\sigma_{2}\right) / 2 C^{\prime},
$$

where the value

$$
\sigma_{2}^{(m e)}=6 \sqrt{3} \delta_{1}\left(m_{x}^{2}-m_{y}^{2}\right)
$$

must be interpreted as the magnetomechanical stress (magnetostress), which is linearly related to square of magnetization and magnetostrictive deformation $u_{2}^{(m e)}=\sigma_{2}^{(m e)} / 2 C^{\prime}$.

It should be now clearly specified, that both the magnetic anisotropy energy $K_{u} m_{j}^{2}$ and Zeeman energy $\mathbf{m H} M$ cannot contribute to the magnetomechanical stress, because both energies are not any explicit functions of strains and their partial derivatives in $u_{\alpha}$ are equal to zero. It means that these energies do not contribute to Eq.11 or Hooke's law (Eq.12). Therefore, all theoretical models, which disregard the magnetostrictive strains and derive the magnetomechanical stress from the anisotropy and/or Zeeman energies, are incompatible with both the equilibrium conditions for Gibbs potential and Hooke's law.

Model results: Effect of the isotropic spin-lattice interaction. The isotropic part of spin-lattice interaction manifests itself in a considerable shift of the Curie temperature under the action of hydrostatic pressure; the experimental value of this shift is $\left(d T_{C} / d P\right)=8 \mathrm{~K} / \mathrm{GPa}$ [21]. The shift is caused by the magnetoelastic renormalization of spin exchange parameter:

$$
J(T) \rightarrow J *(T)-2 \delta_{0}(\Delta V / V) / 3
$$

(see Eqs.5 and 7). The volume change accompanies not only the hydrostatic compression, but also the martensitic transformation of the alloy. Therefore, the value of the exchange parameter jumps (according to Eq.14) when the temperature of the specimen during its cooling reaches the MT temperature, $T_{M}$. The jump of the exchange parameter results in an abrupt change of magnetization value in the saturating magnetic field. The magnetoelastic model involves both the conventional temperature dependence of the exchange parameters $J(T)=\zeta\left(T-T_{C A}\right) / T_{C A}$ or $J^{*}(T)=\zeta\left(T-T_{C M}\right) / T_{C M}$ and standard equations for the temperature dependencies of magnetization values in austenitic and martensitic phases:

$$
\begin{aligned}
& y(T)=\tanh \left[\left(T_{C A} / T\right) y(T)\right], \\
& y(T)=\tanh \left[\left(T_{C M} / T\right) y(T)\right] .
\end{aligned}
$$


For the tetragonal martensites with $c<a$, the temperature of ferromagnetic ordering exceeds the MT temperature, and therefore, $T_{C A}$ is a Curie temperature, which can be experimentally determined. The temperature:

$$
T_{C M}=T_{C A}\left[1+2\left(\delta_{0} / \zeta\right)(\Delta V / V) / 3\right]
$$

is a characteristic parameter, which prescribes the temperature dependence of magnetization below the MT temperature.

The experimental value of $d T_{C A} / d P$ (see above) and relationship $\zeta=n k_{B} T_{C A}$ ( $n$ is the number of magnetic atoms in the unit volume, $k_{B}$ is Boltzmann's constant) result in the estimations $\zeta \approx 0.1 \mathrm{GPa}$ and $\delta_{0} \approx-0.4 \mathrm{GPa}[70]$. With these parameters, the best fit between theoretical and experimental temperature dependencies of magnetization is achieved when the values $\Delta V / V=-3 \cdot 10^{-2}$ and $T_{C M} \approx T_{C A}+30 \mathrm{~K}$ are used for computations [88].

A theoretical temperature dependence of magnetization, as computed using Eqs.15 and 16, is presented in Fig. 4 (upper branch) together with experimental values measured in the saturating magnetic field, $H=10 \mathrm{kOe}$. The computations were carried out taking into account the statistical spread of "local" Curie temperatures in the spatially inhomogeneous martensitic state (for more details, see [88]). Fig. 4 demonstrates an excellent agreement between the model results and experimental data.

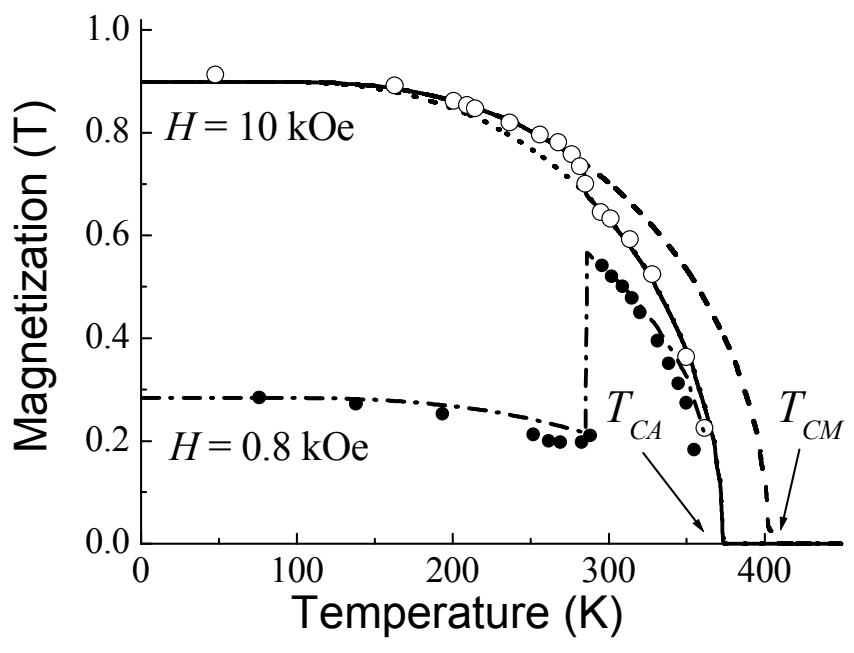

Fig.4. Experimental (open circles) and theoretical (solid line) temperature dependencies of the saturation magnetization obtained for the Ni-MnGa alloy with $c / a=0.94, T_{C}=375 \mathrm{~K}$, and $T_{M}=285 \mathrm{~K}$. The dotted and dashed lines show the magnetization curves computed for the labile phases. The dash-doted line and closed circles show the temperature dependence of magnetization under the external magnetic field $0.8 \mathrm{kOe}$.

It is worth noting, that a five-times-larger value of the magnetoelastic parameter, $\delta_{0} \approx-2 \mathrm{GPa}$, was reported recently [89]. This high value was estimated from the anomalous change of thermal expansion coefficient at the Curie temperature. In this case, the agreement between theoretical and experimental temperature dependencies of magnetization takes place for $\Delta V / V=-6 \cdot 10^{-3}$. Unfortunately, the uncertainty in $\Delta V / V$ values determined by different methods (for more details, see Ref. [90]) makes it difficult to choose between two the $\delta_{0}$ values estimated in Refs. [88] and [89].

Model results: Effects of the anisotropic spin-lattice interaction. An abrupt drop in the magnetization value measured under an external magnetic field of $0.8 \mathrm{kOe}$ accompanies martensitic transformation in $\mathrm{Ni}_{2} \mathrm{MnGa}$ alloy (see the lower branch in Fig. 4). The magnetoelastic model relates this effect to the second term in the energy Eq.6 and explains it as follows: (i) martensitic transformation results in the tetragonal deformation of cubic crystal lattice, which leads to the appearance of a mixture of $x$-, $y$-, and $z$-variants of martensite; (ii) each variant is characterized by a 
magnetic anisotropy constant $K_{\mathrm{u}} \quad($ Eqs.7,8) and an internal anisotropy field $H_{A}=6 \delta M(c-a) / a \sim 10 \mathrm{kOe} \quad[78,88]$, where $\delta=\delta_{1} / M^{2} \approx-23$ is the dimensionless magnetoelastic constant introduced for the sake of convenience; (iii) for the $x$ - and $z$-variants of martensite, the anisotropy field is orthogonal to the external field $H_{y}$, and so, the magnetic moments of these variants are almost perpendicular to the magnetization vector of the $y$-variant when $H<<H_{A}$; (iv) the magnetic anisotropy of the cubic phase is small, and therefore, the direction of the magnetic vector for the whole specimen is close to the direction of the external field, while magnetization is approximately 3 times larger than the magnetization of a tetragonal phase.

In a magnetically non-saturated specimen, the model expression for magnetization is:

$$
M(H, T)=M_{0} y(T, s)\left[(1 / 3)\left(H / H_{D}\right)+(2 / 3)\left(H / H_{S}\right)\right],
$$

where $H_{D} \approx 1 \mathrm{kOe}$ is the demagnetization field and $H_{S} \approx H_{A}=10 \mathrm{kOe}$ is the field of magnetic saturation (for more details, see $[78,82,88]$ ). The theoretical dependence $M(T)$ computed by using Eq. 17 for $H=0.8 \mathrm{kOe}$ agrees with the experimental results (see Fig. 4).

The magnetoelastic model relates the giant field-induced deformation of $\mathrm{Ni}-\mathrm{Mn}-\mathrm{Ga}$ alloy to the field-induced stress defined as the difference $\sigma_{2}^{(m e)}(H)-\sigma_{2}^{(m e)}(0)$. As it was proved in Ref. [73], that the application of a field $\mathbf{H} \| y$ to the martensitic structure depicted in Fig. 3 is physically equivalent to an axial mechanical compression in $y$ direction when the compressive stress is equal to:

$$
\sigma_{y y}^{(e q)}(H)=\left[\sigma_{2}^{(m e)}(H)-\sigma_{2}^{(m e)}(0)\right] / 2 \sqrt{3}
$$

The equivalent stress can be computed using Eqs.5,7,13 and 18. Eqs.5 and 7 result in the linear dependence $m_{x}(H)=H / H_{S}$, where $H_{S}=\left(2 K_{u} / M\right)+\left(D_{y y}-D_{x x}\right) M$, and so, the theoretical field dependence of the stress is quadratic (Eqs.13 and 18).

The quadratic dependence, which was computed for $M\left(H_{S}\right)=0.59 \mathrm{~T}$ and $\mu_{0} H_{S}=0.5 \mathrm{~T}$, is presented in Fig. 5. In the field range $\mu_{0} H_{S}<0.4 \mathrm{~T}$, this dependence agrees with the experimental values of the equivalent stress determined directly from the stress-strain loops measured at different values of the external magnetic field (the details of the experimental procedure resulting in these values can be found elsewhere [73]). The experimental dependencies $M_{x}(H)$ are not linear and the larger the field value, the more pronounced is the deviation from linearity. Due to this deviation, the field dependence of the equivalent stress is far from a quadratic function in the high-field range. Instead, in some cases, the equivalent stress is proportional to the second power of $M_{x}(H)[73,91]$. 


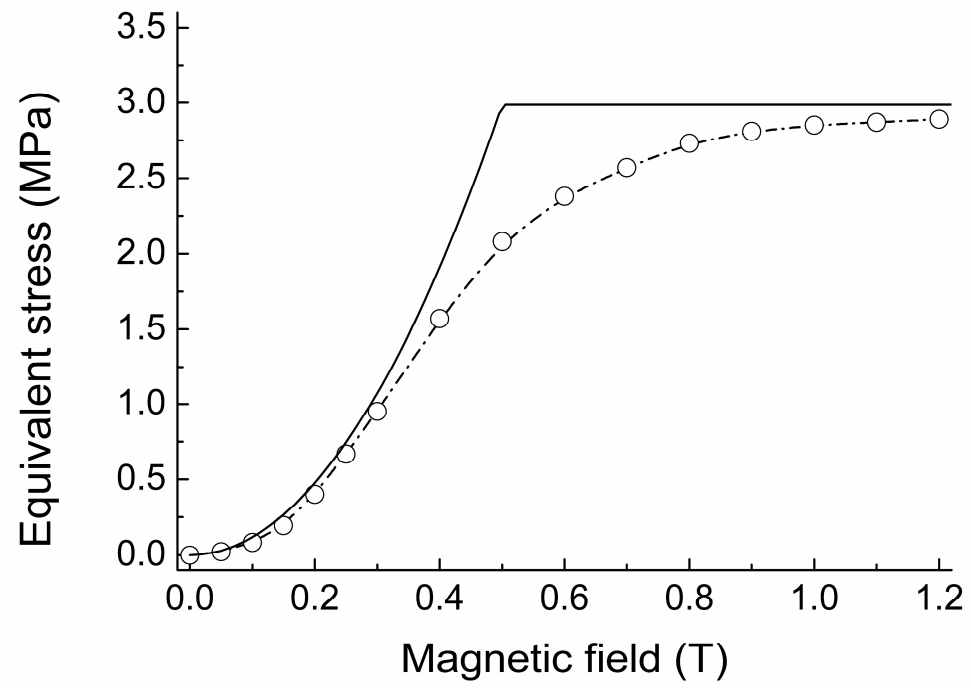

Fig. 5. Equivalent stress found from experimental stress-strain loops [73] (open circles). The solid line represents dependence, which is calculated using Eq.18 and linear field dependence of magnetization.

In those cases, in which the equivalent stress function is known, it can be proved that magnetostrictive deformation is sufficient for the conversion of martensitic twins and to trigger large MFIS. To this end, experimental strain-stress and strain-field dependencies would be used. The strain-stress dependence shows that the variant conversion starts at stress value $\sigma^{(s)} \approx 0.9 \mathrm{MPa}$ and finishes at $\sigma^{(f)} \approx 1.75 \mathrm{MPa}$ (see Fig. 6). Moreover, the final segment of the stress-strain loop, which is an extraction of the elastic part from the total deformation (Fig. 6, Inset), makes it possible to state that the elastic stiffness of martensitic single-variant specimen is

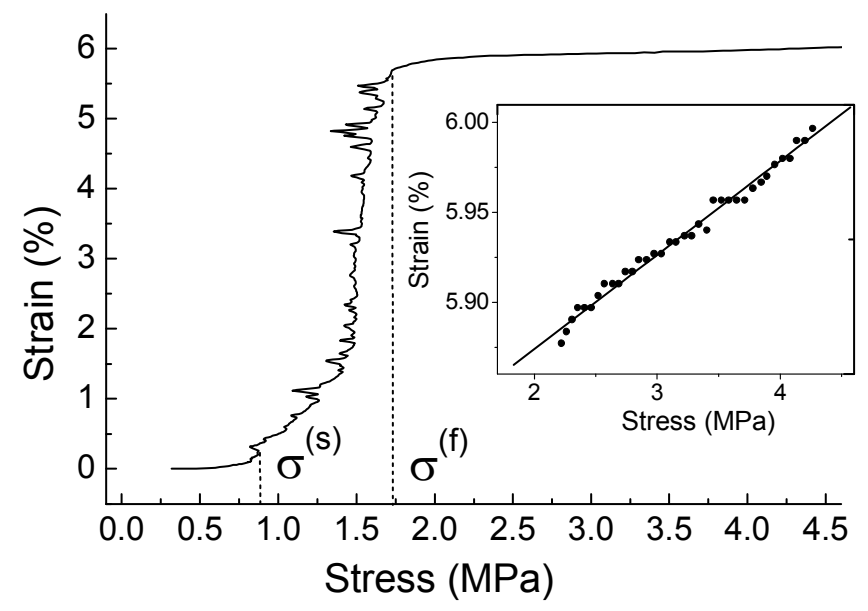

Fig. 6. Experimental strain-stress curve for the $\mathrm{Ni}-\mathrm{Mn}-\mathrm{Ga}$ alloy in the martensitic state [73]. Inset: zoom of the linear part of its final segment. Characteristic stresses indicating the start and finish of variant conversion are denoted as $\sigma^{(\mathrm{s})}$ and $\sigma^{(\mathrm{f})}$, respectively.

$S \approx 1.9 \mathrm{GPa}$. The elastic deformation, which was found in such a way, is shown in Fig. 7 by the straight solid line. The elastic strains values $\varepsilon^{(s)} \approx 4.5 \times 10^{-4}$ and $\varepsilon^{(f)} \approx 9.2 \times 10^{-4}$, corresponding to the start and finish of the variant conversion process are shown by horizontal dashed lines. These strains exceed the value presented in Eq. 1 due to the very low value of stiffness coefficient inherent to the studied alloy. Then, the magnetoelastic strain:

$$
u^{(e q)}(H) \equiv \sigma^{(e q)} / S
$$

is computed using Eqs.13 and 18 and depicted by the solid curve in Fig. 7. The cross points of this curve with the horizontal dashed lines result in the magnetic field values $H^{(s)}=0.27 \mathrm{~T}$ and $H^{(f)}=0.38 \mathrm{~T}$, which must correspond to the characteristic fields of start and finish of magnetically induced twin variant conversion in the specimen. As evidenced from Fig.8, these quantitative predictions have found an experimental confirmation. 

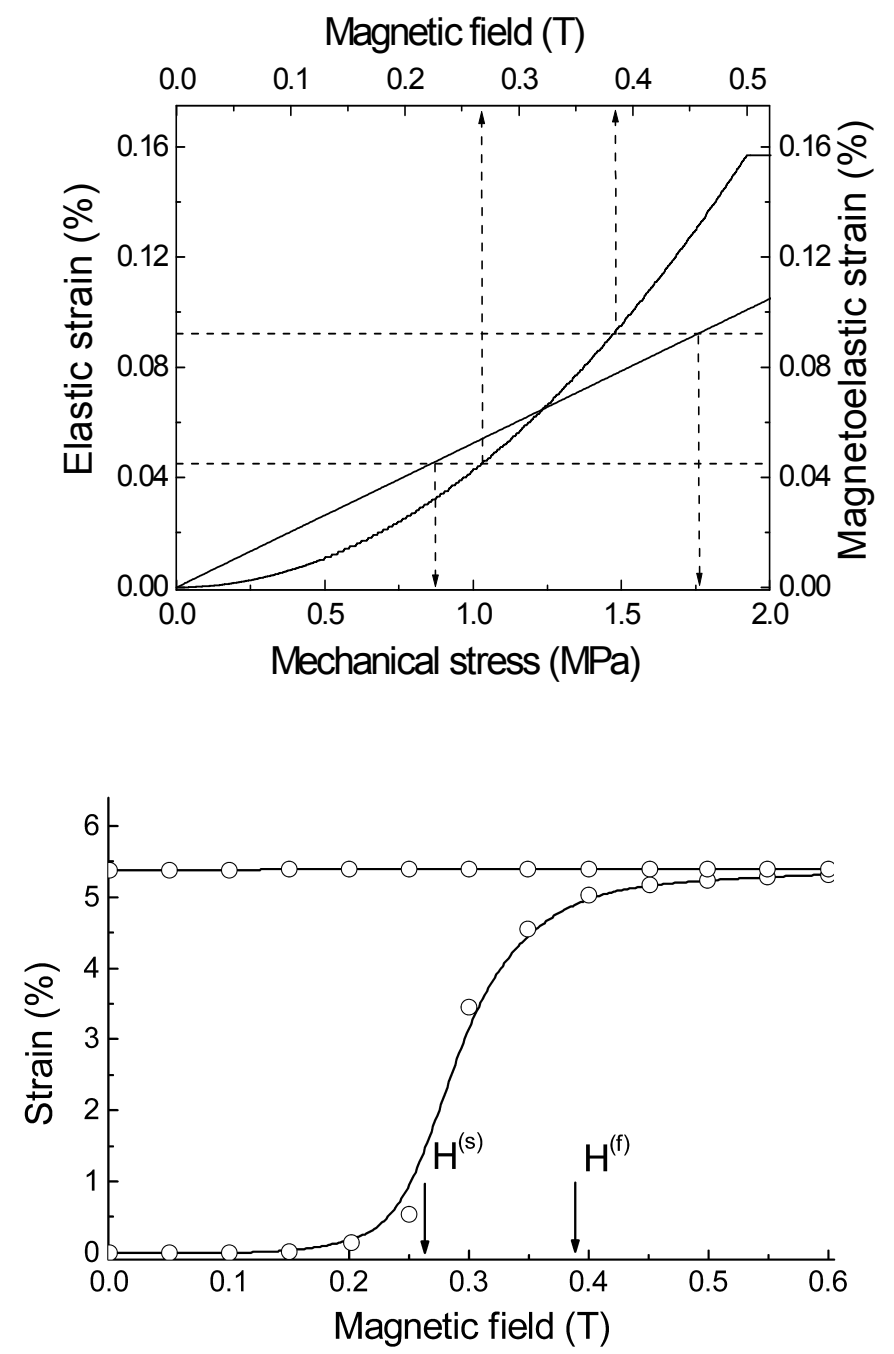

Fig. 7. The elastic part of strain obtained from the final segment of strain-stress loop (straight solid line); stresses and elastic strains corresponding to the start and finish of variant conversion process (down-arrows and horizontal dashed lines, respectively); magnetostrictive strain (solid curve) and magnetic field values (up-arrows) corresponding to the start and finish of magnetically induced deformation accompanying the variant conversion process.
Fig. 8.Giant magnetically induced deformation versus magnetic field of the martensitic single-variant Ni-Mn-Ga sample. The values of characteristic fields determined by using the elastic and magnetostrictive strains in Fig.7 are shown by the arrows. Experimental values (open circles) were obtained by P. Müllner for the same sample as described in Ref.[73].

Fig. 8 depicts the experimental strain-field dependence measured by P. Müllner using the same Ni-Mn-Ga sample studied in Ref.[73]. The values $H^{(s)}$ and $H^{(f)}$ estimated from Fig.7 are shown in Fig. 8 by the arrows. It is seen, that these values are, indeed, very close to the experimental magnetic filed values corresponding to the start and finish of magnetically induced twinningdetwinning cycle in the sample.

The aforementioned experimental results for (magneto)strain obtained on a single-variant martensitic sample are in line with the principle of equivalence between magnetostress and mechanical stress, which follows from the magnetoelastic model (Eq.18, see also Refs.[82,84,86]). Note, that the MFIS and stress-strain tests made on the polyvariant Ni-Mn-Ga sample also support this principle. For instance, it was found in Refs.[59,72] that, experimentally, the maximum strain during compression of the polyvariant specimen is $2 \%$, irrespective of whether magnetic or mechanical force was applied.

The developed magnetoelastic model shows that the magnetoelastic coupling, assumed to be approximately the same in cubic and tetragonal phases, is responsible for the occurrence of magnetostress. The important issue is that the same value of magnetostress may result in different strains in the cubic and martensitic phases, giving rise to the ordinary magnetostriction in the former phase, and with ordinary magnetostriction superimposed on large MFIS in the latter. It becomes clear from the magnetoelastic model that both magnetostriction and large MFIS are caused by magnetoelastic energy Eq. 6 and only the magnetostrictive strain misfit between different components of elastic twins causes the twinning-detwinning cycle and the large MFIS. 


\section{Theoretical resume}

Origin of the magnetically induced stress. The magnetoelastic model states that the giant magnetically-induced deformation of $\mathrm{Ni}-\mathrm{Mn}-\mathrm{Ga}$ martensitic alloys originates from the large magnetostriction inherent to them. The model enables evaluation of both magnetostrictive elastic strain and magnetomechanical stress, which are strictly related to the elastic strain in accordance with Hooke's law. The results presented in Figs. $6-8$ prove that the magnetostrictive elastic strain is sufficiently large for the accomplishment of the twinning/detwinning cycle in the experimental specimen. It should be emphasized, that these results may be reformulated in terms of magnetomechanical stress using Hook's law as expressed by the Eq.19. In contrast, all theoretical models, which disregard magnetoelastic interaction and deduce the magnetomechanical stress from the difference of the magnetic anisotropy energies as:

$$
\sigma^{(m i)}=\frac{K_{u} m_{x}^{2}-K_{u} m_{y}^{2}}{1-c / a}
$$

are not self-consistent because: (i) in the absence of a magnetoelastic interaction, the magnetostrictive strain would be equal to zero so, according to Hooke's law, the stiffness coefficient of an alloy should be equal to infinity in order to provide the finite stress defined in accordance to Eq.20; (ii) the stress tends to infinity when $(1-c / a)$ goes to zero; it means that Eq.20 is obviously not valid in this limit case.

However, the magnetoelastic model "saves" relationship Eq.20 by establishing direct proportionality between $K_{u}$ and $(1-c / a)$ through Eq.8: in this case $(1-c / a)$ vanishes from the formula. That's why this relationship is a reasonable guide for the interpretation of experimental results. For instance, the form of the magnetically induced stress function, which was determined in Ref.[91] by using both Eq.20 and experimental magnetization curves, is correct and close to the function presented in Fig. 5.

Impossibility of a linear strain-field dependence. Magnetoelastic model proves that in the lowfield limit the magnetically-induced stress is a quadratic function of the magnetic field. This conclusion must certainly be highlighted in view of the numerous publications reporting the experimental observation or/and theoretical substantiation of the linear stress - field or strain - field relationships. For example, Eq.2 for magnetostrain given in Ref. [92] yields, in the low-field limit, the relationship:

$$
\varepsilon(H)=M_{S} H /\left(C \varepsilon_{0}\right),
$$

where $M_{\mathrm{S}}$ is the saturation magnetization, $C$ is the elastic modulus and $\varepsilon_{0}$ is the MT strain. Obviously, the strain described by Eq.21 tends to infinity when $\varepsilon_{0}$ tends to zero. Moreover, a linear dependence of giant magnetostrain cannot be correct in principle, because, in reality, the experimentally measured value of deformation is a diagonal component of a symmetric tensor. Should this component be linearly related to the magnetic field, it ought to have a form such as $\varepsilon_{y y}=B_{y} H_{y}$, where $B_{y}$ is a component of some vector, which is independent from the field value (see Fig.3). Let $B_{y}$ be positive, for the sake of definiteness. In this case, the magnetic field applied parallel to $y$-axis $\left(H_{y}>0\right)$ would result in the elongation of the specimen in $y$-direction because $\varepsilon_{y y}>0$, while the field applied in the opposite direction $\left(H_{y}<0\right)$ would result in the contraction of the specimen in the same direction, because in this case $\varepsilon_{y y}<0$. However, this cannot be true for the giant magnetically induced deformation, because for both field directions, this deformation is caused by an increase of the volume fraction of the $y$-variant of martensite. This leads to the 
contraction of the specimen in $y$ direction as follows from Fig 3, where the twin structure with $K_{u}>0$ and $c / a<1$ is depicted.

It is to be concluded therefore, that the development of a self-consistent phenomenological theory of ferromagnetic shape memory effect is possible only with a proper regard to the fundamental principles of magnetoelasticity formulated, e.g., in Refs. [75,76].

\section{Microscopic features of alloys exhibiting giant MFIS}

Naturally, the phenomenological magnetoelastic model is unable to describe directly the process of magnetoplastic yielding of martensite, or dynamic properties or micromechanics of FSMAs. These features are controlled by the twinning dislocations (disconnections) which are the elementary carriers of twin-boundary motion under magnetic field or mechanical force. The dislocation-type microscopic picture of MFIS has been developed by P. Müllner et al. in a number of publications (see, e.g., [35-37,72,86,93]). Alternative modeling of twin interfaces and their motion can be found in Refs.[94-96].

Generally, twinning involves the shear of specific crystallographic planes along specific crystallographic directions. The twinning is always directional in nature which is the main difference as compared to a slip. Fig. 9 shows a schematic of the yielding event due to detwinning caused by the mobile disconnection.

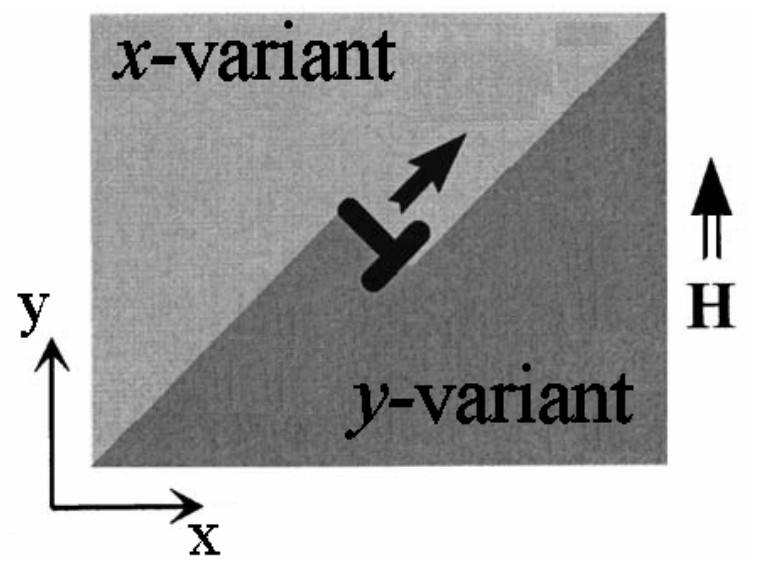

Fig. 9. Schematic of twinning dislocation (disconnection) which moves along a twin boundary under shear stress, $\tau$, and by virtue of its stepwise nature, the twin boundary moves in perpendicular direction thus increasing the volume fraction of the $y$-variant. The thick arrow points the direction of the disconnection movement. The coordinate system, two twin variants and the direction of magnetic field are the same as shown in Fig.3. For more details see text and Ref.[86].

The $x$-variant and $y$-variant are present in a rectangular prismatic sample having all the $\langle 100\rangle$ faces in the cubic phase. These two variants are shown in Fig. 9 by shadings of different intensity. The crystallographic orientation of twins relative to the coordinate system is the same as in Fig. 3. In this configuration, the twin boundary is parallel to the $\{110\}$ plane. In accordance to Eq.18, a magnetic field applied along $y$ direction creates an equivalent compression stress along the same direction whereby the shear stress $\tau=\sigma_{y y}^{(e q)} / 2$ along the twin boundary is exerted. In the configuration shown in Fig. 9, the disconnection starts to move in the direction shown by the arrow when the shear stress becomes larger than some threshold value. By virtue of the stepwise nature of disconnections, the twin boundary moves in a perpendicular direction, thus increasing the volume fraction of the $y$-variant.

Note that the microscopic features of the twin boundary motion just described are in line with the magnetoelastic model. For some more evidences and explanations, let us return to Fig.3. A magnetic field applied in $y$ direction rotates the magnetic moment of the $x$-variant, thus deforms it due to magnetostriction, while leaving the $y$-variant undeformed and its magnetic moment unrotated. Therefore, the resultant lattice misfit between points $A$ and $B$ is estimated as $\Delta l_{A B} \approx \varepsilon^{(m e)} l_{A B} \sim 10^{-4} l_{A B}$. Thus, the lattice misfit exceeds the value $c / 10$ (where $c$ is lattice parameter) when the distance $l_{A B}$ between points $A$ and $B$ (which are considered here as the pinning centers) satisfies inequality $l_{A B}>10^{3} c \sim 0.5 \mu \mathrm{m}$. According to microscopic analysis of $\mathrm{Ni}-\mathrm{Mn}-\mathrm{Ga}$ 
alloys (see, e.g.,[86,97]), the ends of twin boundaries can be pinned at the cores of twinning dislocations situated at the dislocation walls and the distance between the pinning centers may be of the order of $0.5 \mu \mathrm{m}$ or even more. The dimensions of cores are of the order of the lattice parameter, and therefore, according to the Wechsler -Liberman - Read theory [85], the estimated lattice misfit induced by magnetostriction is sufficient for the rearrangement of twinning dislocations and the initiation of a detwinning process.

The distance traveled by the twin boundary should be dependent not only on the applied stress, but also on the spatial distribution and the strength of the pinning centers. The experiment confirms these dependencies [98]. The pinning forces were introduced in the framework of theoretical models of MFIS [83,99]. The following "memorandum" concerning the general physical picture of magnetostrain effect can be found in Ref. [99]:

i) the martensitic state, which exists in the alloy at zero magnetic field may be conventionally considered as the undeformed (and therefore unstressed) reference state;

ii) a magnetic field application results in internal mechanical stressing of the reference state with the stresses being different for the differently oriented martensite variants, and therefore, a microstressed martensitic state arises;

iii) quick partial relaxation of microstresses takes place due to the presence of mobile coherent interfaces, thus the quick magnetoelastic response arises;

iv) the quick relaxation of the field-induced microstresses is not complete because of the imperfectness of the crystal lattice and the incoherent character of some interfaces;

v) the thermal fluctuations of the residual microstresses result in the slow increase of the fieldinduced strains up to saturation.

The imperfection of the crystal lattice and resultant pinning of twin boundaries were described then in a phenomenological way by considering the jump-like displacements of twin boundaries and the statistically-distributed critical stress values, which initiate these displacements [83]. The approach to a theoretical description of MFIS formulated in Ref. [83] is now referred to as the statistical model of the magnetostrain effect. This model enabled the qualitative description of MFIS in the mechanically loaded specimen [73,83] and an explanation of abrupt change of magnetization in the course of twinning-detwinning cycle [84].

Basic idea (v) about the role of thermal fluctuations in the magnetostrain effect was essentially advanced in publications [100-104]. It was shown that thermal fluctuations of internal microstress: a) promote magnetically-induced deformation and cause its slow evolution under the stationary magnetic field [100-103]; b) contribute to the temperature dependence of MFIS [95,103]; c) manifest themselfs in the ultrasonic experiments with martensitic alloys [104].

\section{Summary}

In the present paper, the magnetic properties of ferromagnetic shape-memory alloys and large MFIS produced by the magnetic field-induced twin rearrangement in the martensitic phase are considered. The magnetic/mechanical stress-induced conversion of one crystallographic twin variant into the other is accompanied by a strain, which is close in value to the spontaneous lattice distortion during martensitic transformation. In order to exhibit these effects on a macroscale, materials, such as $\mathrm{Ni}-$ $\mathrm{Mn}-\mathrm{Ga}$ ferromagnetic shape memory alloys, must have particular physical properties as the necessary prerequisites. Then, a sufficient condition is deduced from the balance of these properties. Generally, it can be said that the material must be easily-twinned and mechanically soft martensite with enhanced magnetoelastic interaction. In this context, a lattice instability, low-elastic shear modulus $(\sim 1 \mathrm{GPa})$ and low twinning stress $(\sim 1 \mathrm{MPa})$ of the high magnetostrictive crystal lattice $\left(\varepsilon^{(m e)} \sim 10^{-4}\right.$ ) can be considered as the necessary prerequisites of a large MFIS in Ni-Mn-Ga alloys. The sufficient condition of a large MFIS appears when magnetically-induced equivalent mechanical stress (magnetostress) exceeds by its magnitude the twinning stress.

The main part of the present paper is devoted to the underlying physical mechanism of the magnetostress and giant MFIS in FSMAs. Thermodynamic considerations are used to remove an 
apparent gap between the physics of ordinary magnetostriction and large MFIS. Some key experimental results obtained on the prototype $\mathrm{Ni}-\mathrm{Mn}-\mathrm{Ga}$ FSMA system are considered as a model to illustrate a correspondence with theory.

A magnetostrictive mechanism of the unusual magnetic and magnetomechanical responses observed in the Ni-Mn-Ga alloys is substantiated by a proper using of fundamental concepts such as magnetoelasticity and the commonly known principles of the theory of ferromagnetism and linear elasticity. An agreement between the magnetostrictive mechanism and the crystallographic features of twin rearrangement is demonstrated.

\section{Acknowledgments}

VAC is grateful to Fondazione Cariplo (project 2004.1819-A10.9251) for financial support. The authors are grateful to P. Müllner for the long-term collaboration. An assistance by V.Khovailo and S. Pittaccio in the manuscript preparation is greatly appreciated.

\section{References}

[1] E. du Tremolet de Lacheisserie: Magnetostriction: theory and applications of magnetoelasticity (CRC Press Inc., FL, USA 1993).

[2] H.H. Liebermann and C.D. Graham: Acta Metall. Vol. 25 (1976), p.715.

[3] K. Tajima, Y.Endoh, Y.Ishikawa and W.G. Stirling: Phys.Rev.Lett.Vol.37 (1976), p.519.

[4] M. Sato, B. H. Grier, S. M. Shapiro and H. Miyajima: J. Phys. F: Met. Phys.Vol.12 (1982), p. 2117.

[5] V.V. Kokorin and V.A.Chernenko: Phys. Met. Metall. Vol.65 (1988), p. 190.

[6] T. Kakeshita, K.Shimizu, S. Funada and M.Date: Trans.Jap.Inst.Met.Vol.25 (1984), p.837.

[7] K.Shimizu and T. Kakeshita: ISIJ International. Vol.29 (1989), p.97.

[8] K.Shimizu, N.Yamao, T. Kakeshita, M.Ono, K.Sugiyama and M.Date: Mat.Sci. Forum. Vol.5658 (1990), p.235.

[9] T. Kakeshita, K.Shimizu, T. Maki, I.Tamura, S. Kijima, and M.Date: Scr. Met. Vol.19 (1985), p.973.

[10] V.A. Chernenko: Scr. Mater. Vol. 40 (1999), p. 523.

[11] A.A. Cherechukin, I.E. Dikshtein, D.I. Ermakov, A.V. Glebov, V.V. Koledov, D.A. Kosolapov, V.G. Shavrov, A.A. Tulaikov, E.P. Krasnoperov and T. Takagi: Phys. Lett. A Vol. 291 (2001), p.175.

[12] A. N. Vasil'ev, V. D. Buchel'nikov, T. Takagi, V. V. Khovailo and E. I. Estrin: Phys.-Usp. Vol. 46 (2003), p. 559.

[13] P. Entel, V. D. Buchelnikov, V. V. Khovailo, A. T. Zayak,W. A. Adeagbo, M. E. Gruner, H. C. Herper and E. F. Wassermann: J. Phys. D: Appl. Phys. Vol. 39 (2006), p.865.

[14] V. A. Chernenko, L. Wee, P. G. McCormick and R. Street: J. Appl. Phys.Vol. 85 (1999), p. 7833.

[15] M. R. Ibarra and P. A. Algarabel: Phys. Rev. B.Vol. 50 (1994-II) 41964199.

[16] M. R. Ibarra, C. Marquina, L. Garcia-Orza and A. del Moral: Solid Stat.Commun.Vol. 87 (1993), p. 695.

[17] I.G.Zakrevskiy, V.V.Kokorin and V.A.Chernenko: Soviet Physics Doklady (Proceedings of the Academy of Sciences of the USSR). Vol.34 (1989),p.73. 
[18] R. Kainuma, Y. Imano, W. Ito, Y. Sutou, H. Morito, S. Okamoto, O. Kitakami, K. Oikawa, A. Fujita, T. Kanomata and K. Ishida: Nature.Vol. 439 (2006), p.957.

[19] G.L. Fraga, D.E. Brandao and J.G. Sereni: J.Magn.Magn.Mater. Vol.102 (1991), p.199.

[20] P. J. Webster, K. R. A. Ziebeck, S. L.Town and M. S. Peak: Phil. Mag. B. Vol.49 (1984), p. 295.

[21] V.V.Kokorin and V.A.Chernenko: Phys. Met. Metall. Vol.68 (1989), p.111.

[22] V.A. Chernenko and V.V. Kokorin: Proc. Int. Conf. on Martensitic Transformations (ICOMAT-92, Monterey Inst. for Adv.Studies, Monterey, California, USA,1993), p.1205.

[23] V.A. Chernenko, E.Cesari, V.V. Kokorin and I.N. Vitenko: Scr.Met.et Mat. Vol.33 (1995), p. 1239.

[24] A.N. Vasil'ev, S.A. Klestov, V.V. Kokorin, R.Z. Levitin, V.V. Snegirev and V.A.Chernenko: JETP. Vol.82(3) (1996), p.524.

[25] K. Ullakko, J. K. Huang, C. Kantner, R. C. O’Handley and V. V. Kokorin: Appl. Phys. Lett. Vol. 69 (1996),p. 1966.

[26] S. J. Murray, M. Marioni, S. M. Allen, R. C. O’Handley and T. A. Lograsso: Appl. Phys. Lett. Vol. 77 (2000), p. 886.

[27] O. Heczko, A. Sozinov and K. Ullakko: IEEE Trans. Magn. Vol. 36(2000), p.3266.

[28] A.Sozinov, A.A. Likhachev, N.Lanska and K.Ullakko: Appl.Phys. Lett.Vol. 80 (2002), p.1746.

[29] P.Müllner, V.A.Chernenko and G.Kostorz: J.Appl. Phys. Vol.95 (2004), p.1531.

[30] R. C. O'Handley and S. M. Allen: in Encyclopedia of Smart Materials, edited by M. Schwartz (Wiley, New York 2002).

[31] T. Kakeshita and K. Ullakko: MRS Bull. Vol. 27 (2002), p.105.

[32] E. Cesari, J. Pons, R. Santamarta, C. Segui, and V. A. Chernenko: Archives Metal. Mater. Vol. 49 (2004), p.791.

[33] O. Soderberg, Y. Ge, A. Sozinov, S.-P. Hannula and V. K. Lindroos: Smart Mater. Struct. Vol.14 (2005), p.S223.

[34] V.A. Chernenko and S.Besseghini: Sensors and Actuators A Vol. 142 (2008) pp. 542.

[35] P. Müllner, Z. Clark, L. Kenoyer, W. B. Knowlton and G. Kostorz: Mater. Sci. Eng. A (2007), doi:10.1016/j.msea.2006.12.215

[36] G.Kostorz and P. Müllner: Z. Metallk. Vol.96 (2005), p.703.

[37] P. Müllner: Z. Metallk.Vol.97 (2006), p.205.

[38] T. Sakamoto, T. Fukuda, T. Kakeshita, T.Takeuchi and K. Kishio: Mat. Trans. Vol. 44 (2003), p. 2495.

[39] T. Fukuda, T. Sakamoto, T. Kakeshita, T.Takeuchi and K. Kishio: Mat. Trans. Vol. 45 (2004, p. 188.

[40] F. Gejima, Y. Sutou, R. Kainuma and K. Ishida: Metall. Mater. Trans. Vol. A30 (1999), p.2721.

[41] Y. Sutou, Y. Imano, N. Koeda, T. Omori, R. Kainuma, K. Ishida and K. Oikawa: Appl. Phys. Lett. Vol.85 (2004), p. 4358.

[42] K. Koyama, K. Watanabe, T. Kanomata, R. Kainuma, K. Oikawa and K. Ishida: Appl. Phys. Lett. Vol. 88 (2006)132505. 
[42] T. Krenke, E. Duman, M. Acet, E. F. Wassermann, X. Moya, Ll. Mañosa,A. Planes, E. Suard and B. Ouladdiaf: Phys. Rev.B. Vol. 75 (2007) 104414.

[43] V. K. Sharma, M. K. Chattopadhyay, R. Kumar, T. Ganguli, P.Tiwari and S. B. Roy: J. Phys.: Condens. Matter Vol.19 (2007) 496207.

[44] K. Oikawa, L. Wulff, T. Iijima, F. Gejima, T. Ohmori, A. Fujita, K. Fukamichi, R. Kainuma and K. Ishida: Appl. Phys. Lett. Vol. 79 (2001), p. 3290.

[45] M. Wuttig, J. Li and C. Craciunescu: Scr. Mater. Vol. 44 (2001), p.2393.

[46] V.A.Chernenko, J.Pons, E.Cesari and I.K.Zasimchuk: Scr. Mater. Vol. 50 (2004), p.225.

[47] J. Dadda, H.J. Maier, I. Karaman, H.E. Karaca and Y.I. Chumlyakov: Scripta Mater. Vol. 55 (2006), p. 663.

[48] Z.H. Liu, H.Y. Wang, S.Y. Yu, X.F. Dai, J.L. Chen, G.H. Wu and Y. Liu: Scr. Mater. Vol. 54 (2006), p. 1299.

[49] K. Oikawa, T. Ota, Y. Sutou, T. Ohmori, R. Kainuma and K. Ishida: Mat. Trans. Vol. 43 (2002), p. 2360.

[50] K.Oikawa, Y.Imano, V.A.Chernenko, F.Luo, T.Omori, Y.Sutou, R.Kainuma, T.Kanomata and K.Ishida: Mat. Trans. Vol. 46 (2005), p. 734.

[51] R. Santamarta, J. Font, J. Muntasell, F. Masdeu, J. Pons, E. Cesari and J. Dutkiewicz: Scr. Mater. Vol. 54 (2006), p. 1105.

[52] J. Gutierrez, P. Lazpita, V. Siruguri, J.M. Barandiaran, P. Henry, V.A. Chernenko and T. Kanomata: Int. J. Appl. Electromagn. Mech. Vol. 23 (2006), p. 71.

[53] P.J. Brown, A. P. Gandy, K. Ishida, R. Kainuma, T. Kanomata, H.Morito, K.-U. Neumann, K. Oikawa and K.R.A. Ziebeck: J. Phys.: Cond. Matt.Vol.19(2007) 016201.

[54] E.Cesari, J.Pons, R.Santamarta, C.Segui and V.A. Chernenko: Archives of Metallurgy and Materials Vol. 49, Issue 4 (2004), p. 779.

[55] E.Cesari, J.Pons, C.Seguí and V.A. Chernenko: Applied Crystallography,edited by H. Moraviec and D. Stróz (World Scientific 2004), p. 128.

[56] L. Pareti, M. Solzi, F. Albertini and A. Paoluzi: Eur. Phys. J.Vol. B 32 (2003),p.303.

[57] F. Albertini, A. Paoluzi, L. Pareti, M. Solzi, L. Righi, E. Villa, S. Besseghini and F. Passaretti: J. Appl. Phys. Vol. 100 (2006) 023908.

[58] J.F. Duan, P. Huang, H. Zhang, Y. Long, G.H. Wu, R.C. Ye, Y.Q. Chang and F.R. Wan: J. Alloys and Comp. Vol. 441 (2007), p. 29.

[59] P.Müllner, V.A.Chernenko and G.Kostorz: Scr. Mater. Vol. 49 (2003), p.129.

[60] I.Suorsa, E. Pagounis and K. Ullakko: Appl. Phys.Lett. Vol. 84(2004), p.4658.

[61] L. Straka and O. Heczko: J. Magn.Magn. Mater. Vol. 290-291 (2005), p. 829.

[62] A. Planes and L1. Manosa: Sol.State Phys. Vol.55 (2001), p.159.

[63] X.W. Zhou, J.Li and M. Wuttig: Phys.Rev.B. Vol.44 (1991-II), p.10367.

[64] S. Muto, R. Oshima and F.E. Fujita: Acta Met.Mat. Vol.38 (1990), p.685.

[65] V.A. Chernenko, J.Pons, C.Segui and E.Cesari: Acta Mater.Vol. 50 (2002), p.53.

[66] M. Stipcich, Ll. Mañosa, A. Planes, M. Morin, J. Zarestky, T. Lograsso and C. Stassis: Phys. Rev.B Vol. 70 (2004) 054115. 
[67] J. I. Pérez-Landazábal, V.Sánchez-Alarcos, C.Gómez-Polo, V.Recarte and V. A. Chernenko: Phys. Rev. B Vol. 76 (2007) 092101.

[68] A. Zheludev, S.M. Shapiro, P. Wochner and L.E. Tanner: Phys.Rev. B. Vol. 54 (1996), p. 15045.

[69] L.Manosa, A.Gonzales-Comas, E.Obrado, A.Planes, V.A.Chernenko, V.V.Kokorin and E. Cesari: Phys.Rev.B Vol.55 (1997 - I), p.11068.

[70] V.A. Chernenko, V.A. L'vov, M.Pasquale, C.P.Sasso, S.Besseghini and D.A. Polenur: Int.J.Appl. Electromagn. Mech. Vol. 12 (2000), p. 1.

[71] M. Pasquale, C.P.Sasso, S.Besseghini and V.A.Chernenko: IEEE Trans. Magn. Vol.37 (2001), p.2669.

[72] P.Müllner, V.A.Chernenko, M.Wollgarten and G.Kostorz: J.Appl. Phys. Vol. 92 (2002), p. 6708.

[73] V.A.Chernenko, V. A. L'vov, P.Müllner, G.Kostorz and T.Takagi: Phys. Rev. B Vol.69 ( 2004)134410.

[74] V.V. Martynov and V.V. Kokorin: J.Phys. III France Vol. 2 (1992), p.739.

[75] A. I. Akhiezer, V. G. Bar'yakhtar and S. V. Peletminskii: Spin Waves (Amsterdam: North Holland, 1968).

[76] V. G. Bar'yakhtar and E. A. Turov: Spin Waves and Magnetic Excitations 2 (Elsevier Sci. Publ., Amsterdam Vol. 7,1988, p. 333).

[77] L. Dai, J. Cullen and M. Wuttig: J. Appl. Phys. Vol. 95 (2004), p. 6957.

[78] V. A. L'vov, E. V. Gomonaj and V. A. Chernenko: J. Phys.: Cond. Mat. Vol. 10 (1998), p. 4587.

[79] E. V. Gomonaj and V. A. L'vov: Met. Phys. Mod. Tekhn. Vol. 20 (1998),p. 22.

[80] V. A. Chernenko, V. A. L'vov and E. Cesari: J.Magn.Magn.Mater. Vol. 196 - 197 (1999), p. 859.

[81] V. A. Chernenko, V. A. L'vov, E. Cesari and P. McCormick: Mat. Trans. JIM Vol. 8 (2000), p. 928.

[82] V. A. L'vov, S. P. Zagorodnyuk and V. A. Chernenko: Eur. Phys. J. Vol. B27(2002), p. 55.

[83] N. I. Glavatska, A. A. Rudenko, I. N. Glavatskiy and V. A. L'vov: J.Magn.Magn.Mater. Vol. 265 (2003), p. 142.

[84] M. Pasquale, C. Sasso, G. Bertotty, V.A. L'vov, V. A. Chernenko and A. De Simone: J. Appl. Phys. Vol. 93 (2003), p. 8641.

[85] D. S. Liberman, T. A. Read and M. S. Wechsler: J. Appl. Phys.Vol. 28(1957), p. 532.

[86] P. Müllner, V.A. Chernenko and G. Kostorz: J. Magn. Magn. Mater. Vol. 267 (2003), p. 325.

[87] A. N. Vasil'ev, A. D. Bozhko, V. V. Khovailo, I. E. Dikshtein, V.G. Shavrov, V. D. Buchel'nikov, M. Matsumoto, S. Suzuki, T. Takagi and J. Tani: Phys. Rev. Vol. B59 (1999), p. 1113.

[88] V. A., Chernenko, V. A. L'vov, S. P. Zagorodnyuk and T. Takagi: Phys. Rev.B Vol.67 (2003) 064407.

[89] V.D. Buchelnikov, V.V. Khovailo and T. Takagi: J.Magn.Magn.Mater.Vol. 300 (2006), p.459.

[90] V.A. Chernenko and V.A. L'vov: Phil. Mag. Vol. A73 (1996), p. 999.

[91] L. Straka and O. Heczko: IEEE Trans. Mag. Vol. 39 (2003), p. 3402. 
[92] R. C. O'Handley, S. J. Murray, M. Marioni, H. Nembach and M. S. Allen: J. Appl. Phys. Vol. 87 (2000), p. 4712.

[93] P.Müllner, D. Mukherji, M.Aguirre, R. Erni and G. Kostorz: Solid-to-Solid Phase Transformations in Inorganic Materials 2005, edited by J. M. Howe, D.E. Laughlin, J. K. Lee, U. Dahmen and W.A. Soffa. Vol.2 (TMS, The Minerals, Metals \& Materials Society 2005), p.171.

[94] D. I. Paul, R. C. O’Handley and B. Peterson: J. Appl. Phys.Vol. 97(2005), 10 M312.

[95] R. C. O’Handley, D. I. Paul, S. M. Allen, M. Richard, J. Feuchtwanger, B. Peterson, R. Techapiesancharoenkij, M. Barandiaran and P. Lázpita: Mat. Sci. Eng.A Vol 438-440 (2006), p. 445.

[96] D. I. Paul, W. McGehee, R. C. O’Handley and M. Richard: J.Appl. Phys. Vol.101 (2007), 123917.

[97] V.A.Chernenko, P.Müllner, M.Wollgarten, J. Pons and G.Kostorz: J. de Physique IV France V.112 (2003), p. 951.

[98] M.A. Marioni, S. M. Allen and R. C. O’Handley: Appl.Phys.Lett. Vol.84 (2004), p.4071.

[99] N. I. Glavatska, A. A. Rudenko and V. A. L'vov: J.Magn.Magn.Mater. Vol. 241 (2002), p. 287.

[100] V. A. L'vov, A. A Rudenko and N. I. Glavatska: Phys. Rev. Vol. B71 (2005) 024421.

[101] S. I. Bozhko, V. D. Buchelnikov, S. V. Taskaev, T. Takagi and A. N. Vasil'ev: Int. J. Appl. Electromagn. Mech. Vol. 21 (2005), p. 11.

[102] V. A. L'vov, N. I. Glavatska, I. N. Glavatskiy, Y. Ge, O. Heczko, O. Söderberg and S.-P. Hannula: Int. J.. Appl. Electromagn. Mech. Vol. 23 (2006), p. 75.

[103] N. I. Glavatska, V. A. L'vov and I. N. Glavatskiy: J.Magn.Magn.Mater. Vol. 309 (2007), p. 244.

[104] V. A. L’vov, S. Kustov and E. Cesari: Acta Mater. Vol. 56 (2008), p. 802. 